\title{
Rapid Carbapenem Inactivation Method (rCIM): A New Phenotypic Method for Rapidly Detecting Carbapenemase-Producing Enterobacterales
}

\section{Yameng Liu}

Jinzhou Medical University

Liang Luan

PLA North Military Command Region General Hospital

Jing Liu

PLA North Military Command Region General Hospital

Nan Wan ( $\triangle$ wannan@outlook.com )

PLA North Military Command Region General Hospital

Research article

Keywords: rCIM, carbapenem-resistant Enterobacterales, carbapenemase, mCIM, CNPt-Direct test

Posted Date: August 18th, 2020

DOI: https://doi.org/10.21203/rs.3.rs-58147/v1

License: (c) (i) This work is licensed under a Creative Commons Attribution 4.0 International License.

Read Full License 


\section{Rapid carbapenem inactivation method (rCIM): A new}

2 phenotypic method for rapidly detecting

\section{3 carbapenemase-producing Enterobacterales}

4 Yameng Liu ${ }^{1}$, Liang Luan², Jing Liü and Nan Wan ${ }^{2 *}$

5 Abstract

6 Background: Rapid and accurate methods for detecting carbapenemase-producing

$7 \quad$ Enterobacterales (CPE) are essential for improving patient prognosis and preventing

8 the spread of these microbes. In this study, 103 carbapenem-resistant

9 Enterobacterales (CRE) isolates were collected from clinical specimens; of these, 55

10 CRE isolates were included in the retrospective analysis, and 48 CRE isolates were

11 included in the prospective evaluation. Using sequencing results as the gold standard,

12 we evaluated the performance of the rapid carbapenem inactivation method (rCIM)

13 for detecting carbapenemases in comparison with the modified carbapenem

14 inactivation method (mCIM) and CNPt-Direct test. In rCIM, the test isolate was

15 incubated with meropenem (MEM) disks, and the supernatant obtained via

16 centrifugation was incubated with the indicator strain Escherichia coli ATCC 25922.

17 Growth of the indicator strain was monitored using a nephelometer.

$18 *$ Correspondence: wannan@outlook.com

$19{ }^{1}$ Post Graduate Training Base of General Hospital of Northern Theater Command, Jinzhou

20 Medical University, Jinzhou, Liaoning Province, China

21 2Department of Clinical Laboratory, General Hospital of Northern Theater Command, Shenyang,

22 Liaoning Province, China 
Results: The cut-off value of $\mathrm{rCIM}$ was $0.50 \mathrm{McFarland}$ units. In retrospective analysis, the percent positive agreement and percent negative agreement of rCIM for detecting carbapenemase were $97.1 \%$ and $100 \%$, respectively, and these values were higher than those for mCIM and the CNPt-Direct test. In the prospective evaluation, rCIM was linked to a sensitivity and specificity of $96.4 \%$ and $95 \%$, respectively. Conclusion: rCIM may be a rapid $(<3 \mathrm{~h})$, economical, simple, and reliable method for screening CPE isolates, and it is expected to be routinely implemented in clinical microbiology laboratories.

Keywords: rCIM, carbapenem-resistant Enterobacterales, carbapenemase, mCIM,

CNPt-Direct test

\section{Background}

Enterobacterales comprises a large group of gram-negative bacilli with similar biological characteristics, and these microbes are common causes of hospital-acquired and community-acquired infections [1]. Carbapenems are the last line of defense against Enterobacterales infections. The worldwide emergence and spread of carbapenem-resistant Enterobacterales (CRE) represent a public health threat [2-3]. The carbapenem resistance mechanisms include the production of carbapenemase, high efflux pumps expression, and production of extended-spectrum $\beta$-lactamase (ESBL) and/or AmpC $\beta$-lactamase in combination with mutations that decrease the permeability of the bacterial cell to the entry of carbapenems, but carbapenemase production is the main mechanism [4-5]. Studies have revealed that carbapenemase-producing Enterobacterales (CPE) has higher toxicity, easier 
transmission, poorer prognosis, and higher mortality than non-carbapenemase-producing Enterobacterales (NPE) [6]. Therefore, the early and accurate detection of $\mathrm{CPE}$ is extremely important for infection control and for epidemiologic and therapeutic purposes.

Although molecular methods remain the gold standard [7-8], they are complex and costly, their use is limited by the targets used in the test, and they are not accessible to all microbiology laboratories worldwide. Therefore, phenotype-based assays are urgently needed for carbapenemase screening in clinical microbiology laboratories. At present, several phenotypic methods are available for detecting carbapenemase, including MALDI-TOF carbapenem hydrolysis assays [7], biochemical methods (Carba NP) [7], immunological tests (RESIST-3 O.K.N., NG-Test CARBA 5) [9-10], growth-based assays (modified Hodge test), and the modified carbapenem inactivation method (mCIM) [7]. The growth-based assays require at least 18-24 h, which delays detection. The MALDI-TOF carbapenem hydrolysis assays, immunological methods, and Carba NP test require only 15 min to $4 \mathrm{~h}$ to obtain results. Because of the high cost of mass spectrometers, MALDI-TOF assays cannot be widely implemented in all microbiology laboratories. The immunological methods only target the 3-5 most common carbapenemases. The Carba NP test interprets the result visually based on color changes, and the result is greatly affected by the personnel who judge the results, which can easily lead to false-negative or false-positive results.

6 Carbapenem inactivation method (CIM)-based tests have attracted widespread 
interest in clinical microbiology since 2015, and many studies have described the simplicity, low cost, and high sensitivity and specificity of CIM assays for the detection of CPE [11-14]. CIM assesses the growth of the indicator strain Escherichia coli ATCC 25922 around meropenem disk that were previously incubated with the test isolate. If meropenem in the disk is hydrolyzed by carbapenemase expressed by the test organism, E. coli ATCC 25922 will grow to the edge of the disk or exhibit a diminished zone of growth inhibition. Conversely, a zone of growth inhibition indicates that MEM in the disk is active and that the test isolate does not produce a carbapenemase. mCIM was developed by Dr. Sanchita Das and colleagues (unpublished work) both to overcome the limited sensitivity of CIM for certain enzyme classes and increase the simplicity of the method (purchasing $2 \mathrm{~mL}$ of TSB broths versus aliquoting $400 \mu \mathrm{L}$ of water). In 2017, the Clinical and Laboratory Standards Institute (CLSI) recommended mCIM as the phenotype test for detecting carbapenemase [15-16]. In 2018, CLSI also added mCIM and ethylenediaminetetraacetic acid (EDTA)-CIM into as standard microbial susceptibility tests for screening carbapenemases and differentiating serine enzymes [17].

In this study, we introduced the rapid carbapenem inactivation method (rCIM), a new variant of CIM, and judged whether the test isolate produced carbapenemase by measuring the McFarland index of the indicator strain ATCC 25922. The method shortens the detection time to $2-2.5 \mathrm{~h}$. In this study, we compared rCIM with sequencing and other phenotype tests (mCIM and CNPt-Direct) to evaluate its performance and provided evidence for clinical treatment and the prevention of 
nosocomial infections.

\section{Results}

\section{Establish ment of the rCIM positive cut-off value}

In the positive control, the MEM disks were inactivated, indicating that the growth of the indicator strain ATCC 25922 was unhindered. The median (min-max) baseline-subtracted values at 1.5 and $2 \mathrm{~h}$ were $0.84(0.79-0.92)$ and $1.33(1.31-1.37)$ McFarland units, respectively. In the negative control, the growth of ATCC 25922 was inhibited by MEM disks, and the median values (min-max) at 1.5 and $2 \mathrm{~h}$ were 0.15 $(0.13-0.18)$ and $-0.02(-0.04-0.01)$ McFarland units, respectively. There was no obvious growth difference between the positive and negative controls before $1.5 \mathrm{~h}$, whereas a significant difference was identified after $1.5 \mathrm{~h}$. According to the calculation rule of cut-off value, we established $0.50 \mathrm{McFarland}$ units as the $\mathrm{rCIM}$ positive cut-off value, which was 3 -fold higher than the median negative control at $1.5 \mathrm{~h}$, and evaluated the value using a retrospective and prospective collection of 103 CRE isolates.

\section{Retrospective analysis}

In the retrospective analysis, the 55 organisms and their sequencing and phenotypic methods results are presented in Table 3. The growth of the indicator strain is presented in Figure 1, and the results indicated an apparent growth difference between the CPE and NPE isolates. At $1.5 \mathrm{~h}$, rCIM accurately detected 31/35 CPE isolates, and the median (min-max) was $1.92(0.22-2.50)$ McFarland units. In addition, the value did not reach 0.5 McFarland units $(0.44,0.38$, and 0.49 , respectively) for three $E$. 
111 cloacae isolates at $1.5 \mathrm{~h}$, but the values all exceeded 0.50 McFarland units $(1.10,0.78$,

112 and 0.88 , respectively) at $2 \mathrm{~h}$. At $2 \mathrm{~h}$, the majority of isolates (34/35) had reached 0.50

113 McFarland units with the median (min-max) reaching 2.47 (0.46-3.06). One blaNDM

114 E. cloacae isolate grew to 0.46 McFarland units at $2 \mathrm{~h}$. rCIM detected 20/20 NPEs,

115 and the median values (min-max) at 1.5 and $2 \mathrm{~h}$ were $0.03(-0.10-0.17)$ and 0.05

$116(-0.12-0.15)$ McFarland units, respectively. In the retrospective analysis, the percent

117 positive and negative agreement of rCIM for detecting CPE were $97.1 \%$ and $100 \%$,

118 respectively, and $\kappa$ was 0.961 .

119 In the retrospective analysis, mCIM accurately detected 32/35 CPEs and 20/20

120 NPEs, whereas false-negative results were obtained for three isolates (blaKPC $K$.

121 pneumoniae, blaNDM E. coli, and blaNDM E. cloacae). The percent positive

122 agreement, percent negative agreement, and $\kappa$ of $\mathrm{mCIM}$ were $91.4 \%, 100 \%$, and

123 0.886, respectively. CNPt-Direct accurately detected 33/35 CPEs and 20/20 NPEs,

124 including two false-negative results (blaKPC K. pneumoniae and blaIMP K. oxytoca),

125 and the percent positive agreement, percent negative agreement, and $\kappa$ were $94.3 \%$,

$126 \quad 100 \%$, and 0.923.

127 Prospective evaluation

128 The sequencing, rCIM, mCIM, and CNPt-Direct results for 48 organisms are

129 presented in Table 4. The growth of the indicator strain E. coli ATCC 25922 is shown

130 in Figure 2. In total, 27/28 CPE isolates were detected by rCIM, and the median

131 values (min-max) at 1.5 and $2 \mathrm{~h}$ were $1.32(-0.07-1.71)$ and $1.97(-0.05-2.39)$

132 McFarland units, respectively. A false-negative result was obtained for isolate of 
blaNDM P. mirabilis, reaching -0.05 McFarland units at 2 h. rCIM detected 19/20 NPEs, with the median values (min-max) at 1.5 and $2 \mathrm{~h}$ reaching $0.03(-0.15-0.55)$ and $0.06(-0.12-2.16)$ McFarland units, respectively. A false-positive result was recorded for one PCR-negative K. pneumoniae isolate, and the values at 1.5 and $2 \mathrm{~h}$ were 0.55 and $2.16 \mathrm{McFarland}$ units, respectively. In the prospective evaluation, the sensitivity, specificity, positive predictive value, negative predictive value, and $\kappa$ of rCIM were $96.4 \%, 95 \%, 96.4 \%, 95 \%$, and 0.914 , respectively.

In the prospective evaluation, mCIM accurately detected 18/28 CPE and 19/20 NPE isolates, and false-negative results were obtained for 10 isolates (three $K$. pneumoniae, two E. coli, three E. cloacae, one P. mirabilis, and one E. aerogenes isolate). One PCR-negative K. pneumoniae isolate had an inhibition zone diameter of $6 \mathrm{~mm}$, which denoted a false-positive result. The sensitivity, specificity, positive predictive value, negative predictive value, and $\kappa$ of $\mathrm{mCIM}$ were $64.3 \%, 95 \%, 94.7 \%$, 65.5\%, and 0.557, respectively. CNPt-Direct accurately detected 23/28 CPE and 19/20 NPE isolates, whereas false-negative and false-positive results were recorded for five isolates (two K. pneumoniae, one E. coli, one P. mirabilis, and one E. aerogenes isolate) and one isolate (PCR-negative $K$. pneumoniae), respectively. CNPt-Direct had a sensitivity, specificity, positive predictive value, negative predictive value, and $\kappa$ of $82.1 \%, 95 \%, 95.8 \%, 79.2 \%$, and 0.750 , respectively. The sensitivity and consistency of rCIM were better than those of the other phenotypic tests (mCIM and CNPt-Direct), but there was no difference among the groups concerning specificity. 


\section{Discussion}

Carbapenemase-encoding genes are mostly located in mobile genetic elements such as bacterial plasmids, integrants, and transposons, and they can easily spread among bacteria when the outer membrane is damaged and/or permeability increases, thereby explaining the widespread spread of carbapenem-resistant Enterobacteriaceae isolates globally [18-19]. Therefore, a method for rapidly and effectively detecting carbapenemase is urgently required. In this study, we evaluated a new phenotypic method for the rapid detection of carbapenemases. rCIM correctly detected 61/63 CPE and 39/40 NPE isolates, giving an overall sensitivity of $96.8 \%$ and specificity of 97.5\%, which were slightly lower than the values reported by Muntean et al. [20]. These lower results may be related to the insufficient sample size. In general, rCIM is an excellent method for the rapid screening of CPE.

In this study, the CRE isolates were mainly K. pneumoniae (52.4\%), followed by E. coli $(23.3 \%)$ and E. cloacae (15.5\%). The carbapenem resistance mechanisms of $K$. pneumoniae in our hospital were mainly the production of Klebsiella pneumoniae carbapenemase (KPC, 48.1\%), which is consistent with relevant domestic reports [21]. There were two false-negative results and one false-positive result for $\mathrm{rCIM}$ in this study. In the retrospective analysis, one isolate of blaNDM E. cloacae had a growth value of $0.46 \mathrm{McF}$ arland units at $2 \mathrm{~h}$, approaching the positive cut-off value of 0.50 , which may be related to the weaker activity of the carbapenemase. Another isolate of blaNDM $P$. mirabilis reached a value of -0.05 McFarland units at $2 \mathrm{~h}$, and the CNPt-Direct result was also negative, which may be related to the non-expression or 
low expression of carbapenemase. In addition, a false-positive result was obtained for one PCR-negative K. pneumoniae isolate, with the growth of the indicator strain reaching $2.16 \mathrm{McF}$ arland units at $2 \mathrm{~h}$, and the CNPt-Direct result was also positive, probably linked to the limited number of primers used in our study.

In this study, only blaKPC, blaNDM, and blaIMP were detected, and blaVIM- and blaOXA-positive isolates were not found, which was in accordance with the epidemiology of carbapenemase in China [7,22-23]. In the retrospective analysis, three E. cloacae isolates exhibited increased growth to $0.44,0.38$, and 0.49 McFarland units at $1.5 \mathrm{~h}$, respectively, but the growth of the indicator strain further increased to $1.10,0.78$, and $0.88 \mathrm{McF}$ arland units at $2 \mathrm{~h}$, respectively, indicating the importance of the additional $30 \mathrm{~min}$ of incubation. Another isolate of blaNDM $E$. cloacae had an increase in growth to $0.46 \mathrm{McFarland}$ units at $2 \mathrm{~h}$, approaching the cut-off value of $0.50 \mathrm{McF}$ arland units. To reduce the occurrence of false-negative and false-positive results, the test isolate with growth near the positive cut-off value of $0.50 \mathrm{McF}$ arland units at $2 \mathrm{~h}$ needs to be re-tested or further tested using other tests (molecular or immunological methods). In addition, the per-test cost of rCIM is extremely low, requiring only $2.5 \mathrm{~mL}$ of TSB broth and two MEM disks, comparable to the requirements for other phenotypic experiments. All isolates used in our study were obtained from blood plates, and the performance of rCIM when applied to other culture media requires further research.

rCIM, a variant of CIM, is a test for the rapid and accurate detection of carbapenemase. CIM and its variants have higher sensitivity than Carba NP when 
detecting low-activity carbapenemases [20,24-25]. As reported by Caméléna et al. (2018), the CIMPlus test used EDTA as an inhibitor for detecting MBL and phenylboronic acid (PBA) as a class A enzyme inhibitor, and this test can detect carbapenemases at 8 and $20 \mathrm{~h}$ and identify the type of carbapenemase at $20 \mathrm{~h}$. CIMplus had sensitivities of 95.7 and $97.8 \%$ at 8 and $20 \mathrm{~h}$, respectively, and a specificity of $94.4 \%$, and the characterization was correct for $100,96.9$, and $100 \%$ of class A, B, and D enzymes, respectively [26]. According to Bianco et al. (2019), the direct $\beta$-lactamase inactivation method rapidly detects $\beta$-lactamase and carbapenemase from positive blood culture bottles within several hours, and it had a sensitivity of $99 \%$ and specificity of $100 \%$ when detecting KPC, NDM, and oxacillinas e-48 enzymes [27].

The performance of rCIM should be further evaluated using more types of carbapenemase and more diverse bacteria species, such as Acinetobacter and Pseudomonas. Clavulanic acid and tazobactam can effectively inhibit class A carbapenemases, aztreonam has activity against MBL, and cephalosporins and aztreonam can treat infections caused by class D carbapenemase-expressing microbes. Therefore, we can attempt to add EDTA and PBA to identify the type of carbapenemase in the first incubation of rCIM. We can also attempt to directly detect the production of carbapenemase from positive blood culture bottles and provide timely feedback the results to the clinician.

\section{Conclusion}

Our research revealed that $\mathrm{rCIM}$ is a rapid $(2-2.5 \mathrm{~h})$, inexpensive, and reliable 
221 phenotype test that can quantitatively detect all carbapenemases, and its sensitivity

222 and consistency were higher than those of mCIM and CNPt-Direct, making it

223 routinely useful for small microbiology laboratories.

\section{Methods}

\section{Retrospective isolates}

226 Two separate cohorts were included, including a retrospective cohort of 55

227 carbapenem-resistant Enterobacterales isolates and a prospective cohort of 48 clinical

228 isolates. The retrospective isolates were obtained from our hospital between January

2292016 and September 2018 and previously were molecularly characterized (PCR and

230 gene sequencing) to identify carbapenemase genes. The retrospective collection

231 included $35 \mathrm{CPE}$ isolates carrying the following carbapenemase genes: blaKlebsiella

232 pneumoniae carbapenemase (KPC), nine isolates; blaNew Delhi metallo- $\beta$-lactamase

233 (NDM), 23 isolates; blaimipenemase (IMP), two isolates; blaKPC plus NDM, one

234 isolate; and NPE, 20 isolates. The $55 \mathrm{CRE}$ isolates consisted of E. coli $(\mathrm{n}=16)$,

235 Enterobacter cloacae $(\mathrm{n}=11)$, Klebsiella pneumoniae $(\mathrm{n}=22)$, and Klebsiella

236 oxytoca $(\mathrm{n}=6)$.

\section{Prospective isolates}

238 From October 2018 to May 2019, 48 clinical CRE isolates were collected in our

239 hospital for prospective research, including $28 \mathrm{CPE}$ and 20 NPE isolates. The CPE

240 isolates carried the following carbapenemase genotypes: blaKPC, 17 isolates;

241 blaNDM, eight isolates; bla KPC plus NDM, one isolate; and blaNDM plus IMP, two

242 isolates. This collection of $\mathrm{CRE}$ isolates included the following organisms: $K$. 
pneumoniae $(\mathrm{n}=32)$, E. cloacae $(\mathrm{n}=5)$, E. coli $(\mathrm{n}=8)$, E. aerogenes $(\mathrm{n}=1)$, E.

244 asburiae $(\mathrm{n}=1)$, and Proteus mirabilis $(\mathrm{n}=1)$. All isolates were stored at $-80^{\circ} \mathrm{C}$, and

245 after thawing, the isolates were subcultured on a blood agar plate before testing. Each

246 isolate was identified via MALDI-TOF mass spectrometry (Bruker Daltonik GmbH,

247 Bremen, Germany). Carbapenem (imipenem, MEM, and ertapenem) susceptibility

248 testing was performed using the Vitek 2 Compact gram-negative panel (bioMérieux,

249 Marcy 1'Étoile, France) and K-B disk diffusion method and interpreted according to 250 the 2018 CLSI guidelines.

\section{rCIM}

252

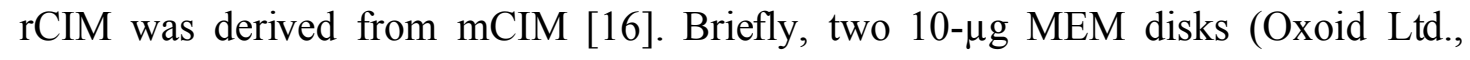
Hampshire, United Kingdom) were added to $1 \mathrm{~mL}$ of distilled water containing two $10-\mu \mathrm{L}$ loopfuls of an overnight isolate, incubated for $30 \mathrm{~min}$ at $35 \pm 2{ }^{\circ} \mathrm{C}$, and centrifuged at $10,000 \mathrm{rpm}$ for $5 \mathrm{~min}$ (high-mucus isolate with a positive mucus filament test needs to be centrifuged twice). The prepared $2.5 \mathrm{~mL}$ of tryptic soy broth (TSB) (Oxoid Ltd.) were taken from a $4^{\circ} \mathrm{C}$ refrigerator and left at room temperature for $30 \mathrm{~min}$, after which a 1.0 McFarland unit suspension of E. coli ATCC 25922 was created. Then, $500 \mu \mathrm{L}$ of the centrifugal supernatant were added, and the mixture was incubated at $35 \pm 2{ }^{\circ} \mathrm{C}$ for $2 \mathrm{~h}$. The growth of the indicator strain $E$. coli was assessed using a nephelometer (bioMérieux) every $30 \mathrm{~min}$, and the McFarland unit results were recorded after subtracting the baseline. For every experiment, the positive and negative controls were $K$. pneumoniae ATCC BAA-1705 and ATCC BAA-1706, respectively. 


\section{Other phenotypic methods}

mCIM was performed following the 2018 CLSI guideline [16-17]. A 1- $\mu \mathrm{L}$ loopful of the CRE isolate was suspended in $2 \mathrm{~mL}$ of TSB containing a 10- $\mu \mathrm{g}$ MEM disk and incubated for $4 \pm 0.25 \mathrm{~h}$ at $35 \pm 2{ }^{\circ} \mathrm{C}$. Subsequently, the MEM disk was removed from the TSB broth at the end of the 4-h incubation using a $10-\mu \mathrm{L}$ inoculation loop and placed on a Mueller-Hinton agar plate freshly plated with a 0.50 McFarland unit suspension of $E$. coli ATCC 25922. Then, the plate was incubated at $35 \pm 2{ }^{\circ} \mathrm{C}$ for 18-24 h. The mCIM result was interpreted according to the inhibition zone, as shown in Table 1.

The CNPt-Direct test was derived from the Carba NP test [28]. Solution A of the CNPt-Direct contains $100 \mu \mathrm{L}$ of $0.05 \%$ phenol red solution consisting of $0.1 \mathrm{mmol} / \mathrm{L}$ $\mathrm{ZnSO}_{4} \cdot 7 \mathrm{H}_{2} \mathrm{O}$ and $0.1 \%$ (vol/vol) Triton X-100 (Solarbio, Beijing, China), and the $\mathrm{pH}$ of solution was adjusted to $7.8 \pm 0.1$. Solution B contains $100 \mu \mathrm{L}$ of solution A supplemented with $12 \mathrm{mg} / \mathrm{mL}$ intravenous (i.v.) imipenem-cilastatin sodium (Merck Pharmaceutical Co., Ltd., Hangzhou, China) immediately before use. Solutions A and B were added to a 96-well microtiter plate (Biotech Engineering Co., Ltd., Shanghai, China), and a $1-\mu \mathrm{L}$ loopful of the test isolate was directly suspended in each well. The 96-well microtiter plate was vortexed for $5-10 \mathrm{~s}$ and then incubated at $35 \pm 2^{\circ} \mathrm{C}$ for 2 h. All isolates were tested in triplicate, and the test was repeated in the case of discrepant results. A color change from red to orange/yellow in the solution B reaction well was interpreted as a positive result. Two independent technicians interpreted the results. In each test, K. pneumoniae ATCC BAA-1705 and ATCC BAA-1706 were 
used as the positive and negative controls, respectively.

\section{PCR detection of carbapenemase genes}

In all retrospective and prospective isolates, DNA was extracted using a bacterial DNA extraction kit (Biotech Engineering Co.), and primers were designed to detect the carbapenemase genes (bla KPC, blaNDM, blaIMP, blaOXA-48-like, and blaVIM), as shown in Table 2. Carbapenemase gene amplification was examined using a PCR kit (Biotech Engineering Co.) with PCR amplification equipment (Hongshi Medical Technology Co., Ltd., Shanghai, China). Briefly, amplification was performed using a protocol consisting of initial denaturation at $94^{\circ} \mathrm{C}$ for $5 \mathrm{~min}$ followed by 36 cycles of denaturation at $94^{\circ} \mathrm{C}$ for $45 \mathrm{~s}$, annealing at $55^{\circ} \mathrm{C}$ for $45 \mathrm{~s}$, and extension at $72^{\circ} \mathrm{C}$ for $1 \mathrm{~min}$, and final elongation at $72^{\circ} \mathrm{C}$ for $10 \mathrm{~min}$. The PCR products were electrophoresed on a $2 \%$ agarose gel at a voltage of 100 for $1 \mathrm{~h}$ using an electrophoresis set (Junyi Dongfang Electrophoresis Equipment Co., Ltd., Beijing, China), and the results were read on a gel imager (Junyi Dongfang Electrophoresis Equipment Co., Ltd.). PCR products were selected and sent to an outside company (Biotech Engineering Co., Ltd.) for sequencing, and sequences were aligned using the BLAST software tool.

\section{Statistical analysis}

The sensitivity, specificity, and positive and negative predictive values of rCIM and other phenotypic tests were calculated using SPSS 22.0 software, and these results were analyzed using the kappa value $(\kappa)$ in comparison with the sequencing results. $\kappa>0.75$ indicated that the two methods are in good agreement, $\kappa=0.4-0.75$ indicated 
moderate agreement, and $\kappa<0.4$ indicated poor agreement.

\section{Abbreviations}

311 PCR: Polymerase Chain Reaction; CLSI: Clinical and Laboratory Standards Institute; CPE:

312 carbapenemase-producing Enterobacterales; CRE: carbapenem-resistant Enterobacterales; mCIM: modified

313 carbapenem inactivation method; rCIM: rapid carbapenem inactivation method; ESBL: extended-spectrum

$314 \quad \beta$-lactamase; NPE: non-carbapenemase-producing Enterobacterales

\section{Acknowledgements}

316 We thank Joe Barber Jr., PhD, from Liwen Bianji, Edanz Editing China (www.liwenbianji.cn/ac), for editing the

317 English text of a draft of th is manuscript.

\section{Authors' contributions}

319 Conceived and designed the study: NW and YL. Con ducted the experiments and analyzed the results: NW, YL, LL,

320 and JL. Drafted the manuscript: YL and NW. All authors contributed to manuscript revision, read and approved the

321 submitted version.

\section{$322 \quad$ Funding}

323 This project was internally funded. External funding bodies had no role in the study design, data collection,

324 analysis, in terpretation or writing of the manuscript.

325 Availability of data and materials

326 The datasets used and/or analysed during the current study are available from the corresponding author on

327 reasonable request.

\section{Ethics approval and consent to participate}

329 Not applicable.

\section{Consent for publication}




\section{Competing interests}

333 The authors declare that they have no competing in terests.

\section{References}

335 1. Duin D, Paterson DL. Multidrug-Resistant Bacteria in the Community: Trends and Lessons Learned. Infect Dis Clin NorthAm. 2016;30:377-390. Med. 2012;27:128-142.

3. Sun K, Xu X, Yan J, Zhang L. Evaluation of Six Phenotypic Methods for the Detection of Carbapenemases in Gram-Negative Bacteria With Characterized Resistance Mechanisms. Ann Lab Med. 201 7;37;305-312. 2016;14:95-108.

BM.

The

Problem

of 2016;54:529-534.

6. Tamma PD, Goodman KE, Harris AD, Tekle T, Roberts A, Taiwo A, et al. Comparing the Outcomes of Patients With Carbapenemase-Producing and Non-Carbapenemase-Producing Carbapenem-Resistant Enterobacteriaceae Bacteremia. Clin Infect Dis. 2017;64:257-264. for Detection of Class A and B Carbapenemase-Producing Enterobacteriaceae in China. J Clin Microbiol. 
8. Dortet L, Fusaro M, Naas T. Improvement of the Xpert Carba-R Kit for the Detection of Carbapenemase-Producing Enterobacteriaceae. Antimicrob Agents Chemother. 2016;60:3832-3837.

9. Glupczynski Y, Jousset A, Evrard S, Bonnin RA, Huang TD, Dortet L, et al. Pros pective evaluation of the OKN K-SeT assay, a new multiplex immunochromatographic test for the rapid detection of OXA-48-like, KPC and NDM carbapenemases. J Antimicrob Chemother. 2017;72:1955-1960.

10. Boutal H, Vogel A, Bernabeu S, Devilliers K, Creton E, Cotellon G, et al. A multiplex lateral flow immunoassay for the rapid identification of NDM-, KPC-, IMP- and VIM-type and OXA-48-like carbapenemase-producing Enterobacteriaceae. J Anti microb Chemother. 201 8;73: 909-915.

11. Zwaluw K, Haan A, Pluister GN, Bootsma HJ, Neeling AJ, Schouls LM. The carbapenem inactivation method (CIM), a simple and low-cost alternative for the Carba NP test to assess phenotypic carbapenemase activity in gram-negative rods. PLoS One 2015;10:e0123690.

12. Gauthier L, Bonnin RA, Dortet L, Naas T. Retrospective and prospective evaluation of the Carbapenem inactivation method for the detection of carbapenemase-producing Enterobacteriaceae. PLoS One 2017; 12:e0170769.

13. Aguirre-Quiñonero A, Cano ME, Gamal D, Calvo J, Martínez-Martínez L. Evaluation of the carbapenem Dis. $2017 ; 88: 214-218$. aeruginosa is olates compared with other methods. J Chemother. 2017;29:144-149. 
16. Laolerd W, Akeda Y, Preeyanon L, Ratthawongiirakul P, Santanirand P. Carbapenemase-Producing Carbapenem-Resistant Enterobacteriaceae from Bangkok, Thailand, and Their Detection by the Carba NP and Modified Carbapenem Inactivation Method Tests. Microb Drug Resi st. 201 8;24:1 006-1011.

17. CLSI. Performance standards for antimicrobial susceptibility testing. 28th ed.Wayne, PA: Clinical and Laboratory Stan dards Institute; 2018.

18. Carattoli A. Plas mids and the spread of resistance. Int J Med Microbiol. 2013;303:298-304.

19. Antunes NT, Lamoureaux TL, Toth M, Stewart NK, Frase H, Vakulenko SB. Class D $\beta$-lactamases: are they all carbapenemases?. Antimicrob Agents Chemother. 2014;58: 2119-2125.

20. Muntean MM, Muntean AA, Gauthier L, Creton E, Cotellon G, Popa MI, et al.. Evaluation of the rapid carbapenem inactivation method (rCIM): a phenotypic screening test for carbapenemase-producing Enterobacteriaceae. J Anti microb Chemother. 2018;73:900-908.

21. Li H, Zhang J, Liu Y, Zheng $\mathrm{R}$, Chen $\mathrm{H}$, Wang $\mathrm{X}$, et al.. Molecular characteristics of carbapenemase-producing Enterobacteriaceae in China from 2008 to 2011: predominance of KPC-2 enzyme. Diagn Microbiol Infect Dis. 2014;78:63-65.

22. Zhang R, Liu L, Zhou H, Chan WE, Li J, Fang Y, et al.. Nationwide Surveillance of Clinical Carbapenem-resistant Enterobacteriaceae (CRE) Strains in China. EBioMedicine. 2017;19:98-106.

23. Zhang Y, Wang Q, Yin Y, Chen H, Jin L, Gu B, et al.. Epidemiology of Carbapenem-Resistant Enterobacteriaceae Infections: Report from the China CRE Network. Antimicrob Agents Chemother. 2018;62:e01882-17.

24. Madkour LA, Sol iman MS, Hassan DM, Soliman NS, ElMahdy YA. Detection of carbapenemase-producers: evaluating the performance of the carbapenem in activation method and Carba NP test versus mul tiplex PCR. J Glob Antimicrob Resist. 201 7; 9:10-14. 
25. Baeza LL, Pfennigwerth N, Greissl C, Göttig S, Saleh A, Stelzer Y, et al.. Comparison of five methods for detection of carbapenemases in Enterobacterales with proposal of a new algorithm. Clin Microbiol Infect. 2019;25: 1286.e9-1286.e15.

26. Caméléna F, Cointe A, Mathy V, Hobson C, Doit C, Bercot B, et al.. Within-a-Day Detection and Rapid Characterization of Carbapenemase by Use of a New Carbapenem Inactivation Method-Based Test, CIMplus. J Clin Microbiol. 2018;56:e0137-18.

27. Bianco G, Boattini M, Iannaccone M, Fossati L, Cavallo R, Costa C. Direct $\beta$-Lactam Inactivation Method: a New Low-Cost Assay for Rapid Detection of Carbapenemase- or Extended-Spectrum- $\beta$-Lactamase-Producing Enterobacterales Directly from Positive Blood Culture Bot tles. J Clin Microbiol. 2019;58:e01178-19.

28. Pasteran F, Tijet N, Melano RG, Corso A. Simp lified Protocol for Carba NP Test for Enhanced Detection of Carbapenemase Prod ucers Directly from Bacterial Cultures. J Clin Microbiol. 201 5;53:3908-391 1.

29. Poirel L, Walsh TR, Cuvillier V, Nordmann P. Mul tiplex PCR for detection of acquired carbapenemase genes. Diagn Microbiol Infect Dis. 2011;70:1 19-123.

Table 1. Interpretation of the modified carbapenem inactivation method (mCIM) result

\section{Zone Size}

\begin{tabular}{ll}
\hline $\mathrm{mCIM}$ to $15 \mathrm{~mm}$ or 16 to & 16 to $18 \mathrm{~mm}$ or $\geq 19 \mathrm{~mm} \geq 19 \mathrm{~mm}$ \\
& $18 \mathrm{~mm}$ with pinpoint $\quad$ with pinpoint colonies \\
colonies in the & in the zone of growth \\
zone of growth & inhibition \\
inhibition & \\
\hline
\end{tabular}




\begin{tabular}{|c|c|c|c|}
\hline \multirow[t]{3}{*}{ Interpretation } & Positive & Indeterminate & Negative \\
\hline & (carbapenemase & & (non-carbapenemase \\
\hline & producer) & & producer) \\
\hline
\end{tabular}

413 Table 2. Primers for the detection of carbapenemase-producing Enterobacterales

\begin{tabular}{|c|c|c|c|}
\hline $\begin{array}{l}\text { Carbapenemase } \\
\text { gene }\end{array}$ & Primer sequences ${ }^{a}\left(5^{\prime}-3^{\prime}\right)$ & $\begin{array}{l}\text { Amplicon } \\
\text { size (bp) }\end{array}$ & Reference \\
\hline \multirow[t]{2}{*}{ blaKPC } & CGTCTAGTTCTGCTGTCTTG & 798 & [29] \\
\hline & CTTGTCATCCTTGTTAGGCG & 232 & \\
\hline \multirow[t]{2}{*}{ blaNDM } & GGTTTGGCGATCTGGTTTTC & 621 & [29] \\
\hline & CGGAATGGCTCATCACGATC & & \\
\hline \multirow[t]{2}{*}{ blalMP } & GGAATAGAGTGGCTTAAYTCTC & 232 & [29] \\
\hline & GGTTTAAYAAAACAACCACC & & \\
\hline \multirow[t]{2}{*}{ blaOXA-48-like } & GCGTGGTTAAGGATGAACAC & 438 & [29] \\
\hline & CATCAAGTTCAACCCAACCG & & \\
\hline \multirow[t]{2}{*}{ blaVIM } & ATCGCAACGCAGTCGTTTGA & 318 & Corporate \\
\hline & CCCGCTCGATGAGAGTCCTT & & synthesis \\
\hline
\end{tabular}

414 The first and second primers for each gene are the forward and reverse primers, respectively. KPC, Klebsiella

415 pneumoniae carbapenemase; NDM, New Delhi metallo- $\beta$-lactamase; IMP, imipenemase; OXA-48-like,

416 Oxacillinase-48-like; VIM, Verona integron-encoded metallo- $\beta$-lactamase.

417 Table 3. Results of rCIM, mCIM, and CNPt-Direct for $55 \mathrm{CRE}$ isolates in the 418 retrospective analysis

\begin{tabular}{llllll}
\hline Organism (n) & Species & No. of & rClM & mClM & CNPt-Direct \\
\hline
\end{tabular}


carbapenemase producer (35)

Class A (9)
KPC (9)
K. pneumoniae
9
$+\quad 8 / 9+$
$8 / 9+$

Class B (25)

$\begin{array}{llllll}\operatorname{NDM}(23) & \text { E. coli } & 8 & + & 7 / 8+ & + \\ & \text { E. cloacae } & 10 & 9 / 10+ & 9 / 10+ & + \\ & \text { K. pneumoniae } & 2 & + & + & + \\ & \text { K. oxytoca } & 3 & + & + & + \\ \operatorname{IMP}(2) & \text { K. oxytoca } & 2 & + & + & 1 / 2+\end{array}$

Coproducers (1)

KPC-NDM (1) K. pneumoniae 1

non-carbapenemase producer (20)

$\begin{array}{lc}\text { E. coli } & 8 \\ \text { E. cloacae } & 1 \\ \text { K. pneumoniae } & 10 \\ \text { K. oxytoca } & 1\end{array}$

419 rCIM, rapid carbapenem inactivation method; mCIM, modified carbapenem inactivation method; CRE,

420 carbapenem-resistant Enterobacterales; KPC, Klebsiella pneumoniae carbapenemase; NDM, New Delhi

421 metallo- $\beta$-lactamase; IMP, imi penemase; + , positive; -, negative.

422 Table 4. Results of rCIM, mCIM, and CNPt-Direct for $48 \mathrm{CRE}$ isolates in the

423 prospective evaluation 


\begin{tabular}{|c|c|c|c|c|c|}
\hline \multirow[t]{2}{*}{ Organism (n) } & \multirow[t]{2}{*}{ Species } & \multicolumn{4}{|l|}{ No. of } \\
\hline & & solates & rCIM & $\mathrm{mCIM}$ & CNPt-Direct \\
\hline \multicolumn{6}{|c|}{ carbapenemase producer (28) } \\
\hline \multicolumn{6}{|l|}{ Class A(17) } \\
\hline \multirow[t]{2}{*}{$\mathrm{KPC}(17)$} & K. pneumoniae & 16 & + & $14 / 16+$ & $14 / 16+$ \\
\hline & E. coli & 1 & + & - & - \\
\hline \multicolumn{6}{|l|}{ Class B (8) } \\
\hline \multirow[t]{5}{*}{ NDM (8) } & E. coli & 1 & + & - & + \\
\hline & E. cloacae & 1 & + & - & + \\
\hline & K. pneumoniae & 4 & + & $3 / 4+$ & + \\
\hline & P. mirabilis & 1 & - & - & - \\
\hline & E. aerogenes & 1 & + & - & - \\
\hline \multicolumn{6}{|l|}{ Coproducers (3) } \\
\hline KPC-NDM (1) & E. cloacae & 1 & + & + & + \\
\hline NDM-IMP (2) & E. cloacae & 2 & + & - & + \\
\hline \multicolumn{6}{|c|}{ non-carbapenemase producer (20) } \\
\hline & E. coli & 6 & - & - & - \\
\hline & E. cloacae & 1 & - & - & - \\
\hline & K. pneumoniae & 12 & 11/12- & $11 / 12-$ & $11 / 12-$ \\
\hline & E. asburiae & 1 & - & - & - \\
\hline
\end{tabular}

424 rCIM, rapid carbapenem inactivation method; mCIM, modified carbapenem inactivation method; CRE, 
Fig. 1. The growth of the indicator strain Escherichia coli ATCC 25922 in the retrospective analysis. The box plot showing the growth of E. coli ATCC 25922 at 1.5 and $2 \mathrm{~h}$ in the retrospective analysis $(\mathrm{n}=55)$. The hollow circles represent the outliers. The horizontal black line at $0.50 \mathrm{McFarland}$ units represents the positive cut-off value. The results are presented as baseline-subtracted McFarland units, and measurements were performed every $30 \mathrm{~min}$.

Fig. 2. The growth of the indicator strain Escherichia coli ATCC 25922 in the prospective evaluation. A box plot presenting the growth of E. coli ATCC 25922 at 1.5 and $2 \mathrm{~h}$ in the prospective evaluation $(\mathrm{n}=48)$. The hollow circles represent the 436 outliers, and the asterisks indicate extreme values. The horizontal black line at 0.50 McFarland units represents the positive cut-off value. The results are presented as baseline-subtracted McFarland units, and measurements were performed every 30 $\min$. 


\section{Figures}

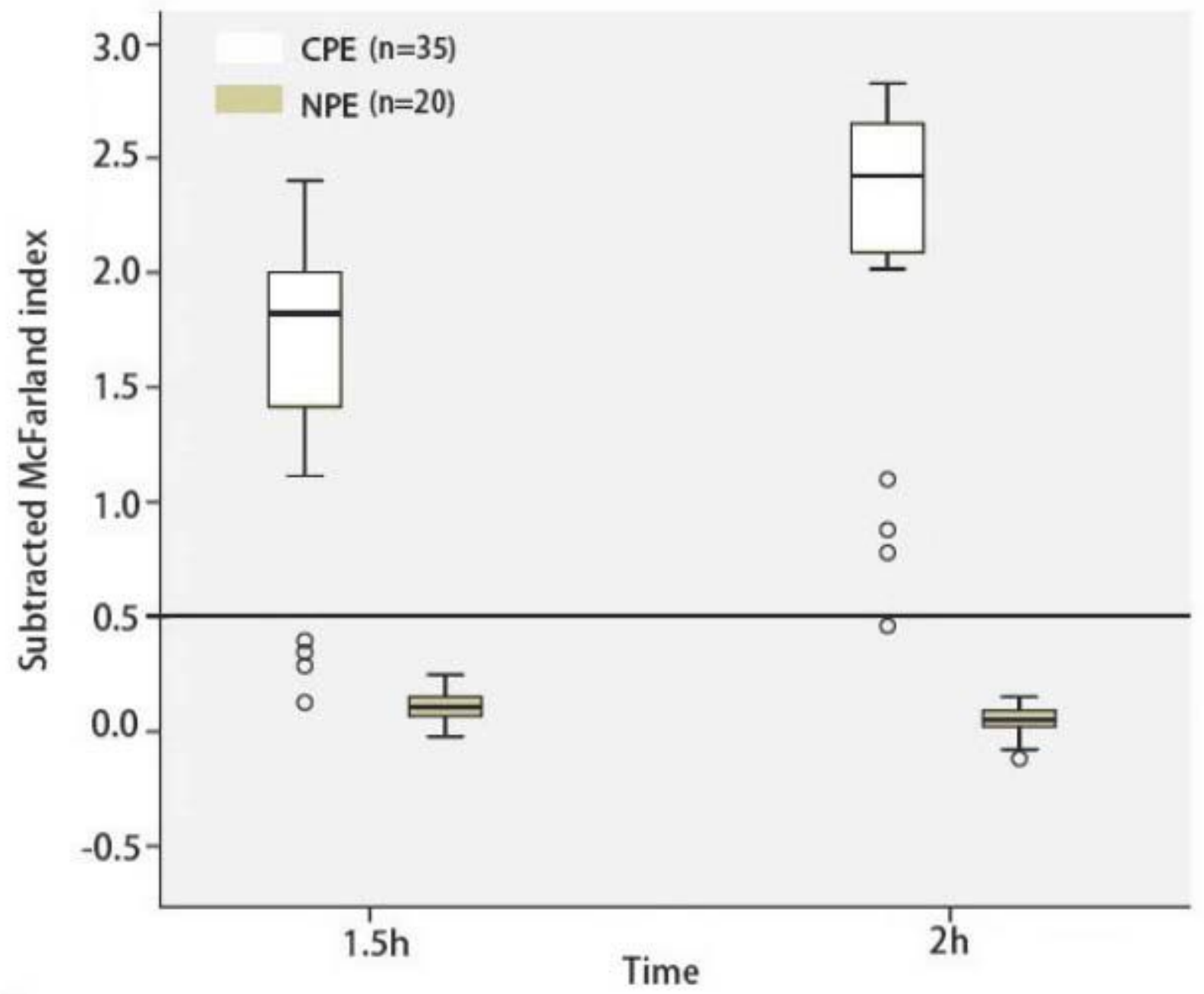

Figure 1

The growth of the indicator strain Escherichia coli ATCC 25922 in the retrospective analysis. The box plot showing the growth of E. coli ATCC 25922 at 1.5 and $2 \mathrm{~h}$ in the retrospective analysis $(n=55)$. The hollow circles represent the outliers. The horizontal black line at $0.50 \mathrm{McF}$ arland units represents the positive cutoff value. The results are presented as baseline-subtracted McFarland units, and measurements were performed every $30 \mathrm{~min}$. 


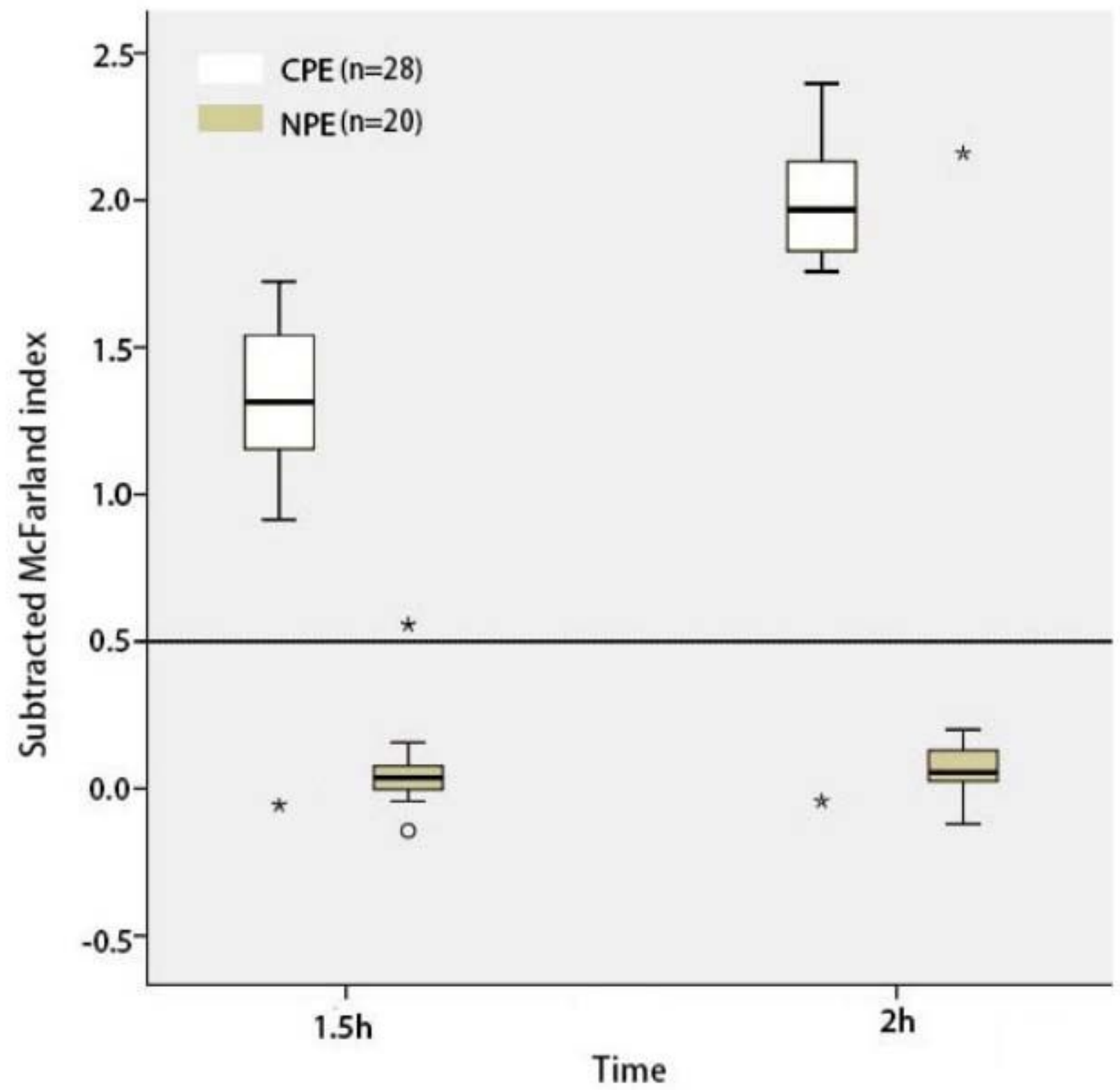

Figure 2

The growth of the indicator strain Escherichia coli ATCC 25922 in the prospective evaluation. A box plot presenting the growth of E. coli ATCC 25922 at 1.5 and $2 \mathrm{~h}$ in the prospective evaluation $(n=48)$. The hollow circles represent the outliers, and the asterisks indicate extreme values. The horizontal black line at $0.50 \mathrm{McF}$ arland units represents the positive cut-off value. The results are presented as baselinesubtracted McFarland units, and measurements were performed every 30 min. 